\title{
ON THE COMPUTATION OF UNIT GROUPS AND CLASS GROUPS OF TOTALLY COMPLEX QUARTIC FIELDS
}

\author{
M. POHST AND J. GRAF V. SCHMETTOW
}

\begin{abstract}
We describe the computation of the unit group and the class group of the 81322 totally complex quartic fields with discriminant less than one million. $45.6 \%$ of those fields have trivial class groups; the maximal class number occurring is 70 .
\end{abstract}

\section{INTRODUCTION}

In [3] we presented computations of the unit group and the class group of all 13073 totally real quartic fields with discriminant below $10^{6}$. In this paper we do the analogous calculations in the totally complex case. Generating equations, integral bases as well as Galois groups $\mathscr{G}$ were again obtained from D. Ford [6]. Since the unit rank is one, the computation of the unit group was much easier this time; on the other hand, the class groups were in general more complicated.

\section{UNIT GROUPS}

The structure of the unit group of a totally complex quartic number field is

$$
\langle\zeta\rangle \times\left\langle\varepsilon_{0}\right\rangle
$$

where $\zeta$ denotes a generator of the torsion subgroup $T U(F)$ and $\varepsilon_{0}$ a fundamental unit. The regulator $2|\log | \varepsilon_{0}||$ is denoted by $R_{F}$. The (cyclic) torsion subgroup was computed by the methods described in [8]. Its order $w$ is at most 12. In detail we found

\begin{tabular}{|l||c|c|c|c|c|c|}
\hline & $w=2$ & $w=4$ & $w=6$ & $w=8$ & $w=10$ & $w=12$ \\
\hline \# of fields & 59964 & 8212 & 13143 & 1 & 1 & 1 \\
\hline
\end{tabular}

It can be easily seen that there is no quartic number field with more than twelve roots of unity and that there is exactly one field for $w=8,10,12$ [8]. These fields are defined by roots of the following polynomials:

$$
\begin{array}{lll}
w=8: & t^{4}+1 & \left(\mathscr{G}=V 4, d_{F}=256, R_{F} \approx 1.763\right) \\
w=10: & t^{4}-t^{3}+t^{2}-t+1 & \left(\mathscr{G}=C 4, d_{F}=125, R_{F} \approx 0.9624\right) \\
w=12: & t^{4}-t^{2}+1 & \left(\mathscr{G}=V 4, d_{F}=144, R_{F} \approx 1.317\right) .
\end{array}
$$

Received by the editor June 21, 1991 and, in revised form, March 11, 1992. 1991 Mathematics Subject Classification. Primary 11Y40, 11-04, 11R16, 11R27, 11 R29. Research supported by Deutsche Forschungsgemeinschaft. 
A fundamental unit $\varepsilon_{0}$ was determined with an algorithm of J. Buchmann [1]. We give below a short description of the essential ideas. Let $F=\mathbb{Q}(\rho)$ be a complex quartic field with ring of integers $o_{F}=\mathbb{Z} \omega_{1}+\cdots+\mathbb{Z} \omega_{4}$ and discriminant $d_{F}$. For each element $\alpha \in F$ there are four conjugates, say $\alpha^{(1)}=\alpha, \alpha^{(2)}$ and the corresponding complex conjugates $\alpha^{(3)}=\overline{\alpha^{(1)}}, \alpha^{(4)}=$ $\overline{\alpha^{(2)}}$. For any fractional ideal a the image $\varphi(\mathbf{a})$ under the mapping

$$
\varphi: \mathbf{a} \rightarrow \mathbf{R}^{2}: \alpha \mapsto\left(\left|\alpha^{(1)}\right|^{2},\left|\alpha^{(2)}\right|^{2}\right)
$$

is a discrete subset of Euclidean 2-space. An element $0 \neq \mu \in \mathbf{a}$ is called minimal if the corresponding norm body

$$
Q(\mu):=\left\{\left.\left(x_{1}, x_{2}\right) \in \mathbf{R}^{2}\left|0 \leq x_{i} \leq\right| \mu^{(i)}\right|^{2}(i=1,2)\right\}
$$

does not contain $\varphi(\alpha)$ for any $\alpha \in$ a different from 0 and $\mu$ modulo the torsion subgroup $T U(F)$. It is easily seen that minimal elements $\mu$ of a have bounded norm [1]:

$$
|N(\mu)| \leq\left(4 / \pi^{2}\right) d_{F}^{1 / 2} N(\mathbf{a}) .
$$

Let $\{i, j\}=\{1,2\}$ be a pair of conjugate directions. An element $\nu$ is called $i$-neighbor of a minimal element $\mu \in \mathbf{a}$ if it is minimal subject to $\left|\nu^{(j)}\right|\left\langle\left|\mu^{(j)}\right|\right.$ and $\left|\nu^{(i)}\right|$ as small as possible. Note that $\nu$ is uniquely determined modulo $T U(F)$ by these properties.

Obviously, 1 is minimal in $o_{F}$. Hence, starting with $\mu_{0}=1$, we obtain a sequence of all minimal elements $\left(\mu_{k}\right)_{k \in \mathbb{Z}}$ of $o_{F}$ in which $\mu_{k+1}$ is the 2neighbor of $\mu_{k}$ and, conversely, $\mu_{k}$ is the 1-neighbor of $\mu_{k+1}$. That sequence is purely periodic, and if $p>0$ is chosen minimal such that $\mu_{p}$ is a unit, then $\mu_{p}$ is a fundamental unit of $F$. From this an algorithm for computing a fundamental unit is almost immediate. We only add a few remarks about the calculation of $i$-neighbors. In general, one proceeds by doubling the range for the $i$ th conjugate and determining all elements in the corresponding norm body. If no element is obtained, that range will be increased again. On the other hand, each time we find a candidate $\mu$ for the next $i$ th neighbor, the conjugates of $\mu$ decrease the bounds for potential further candidates. Since counting lattice points in boxes is in general not very efficient, it is recommended to cover any norm body by a suitable ellipsoid whose lattice points can be determined faster (see $[1,5])$.

Since we cannot present all fundamental units, we conclude this section with a few remarks on the size of the regulators that occur. They vary between 0.337 (discriminant $\left.d_{F}=229\right)$ and $570.2\left(d_{F}=965361\right)$. With respect to the Galois group of the field we get the following distribution:

\begin{tabular}{|r||c|c|c|c|c||r|}
\hline & $\mathrm{C} 4$ & $\mathrm{D} 4$ & $\mathrm{~S} 4$ & $\mathrm{~A} 4$ & $\mathrm{~V} 4$ & $\#$ \\
\hline \hline & 54 & 36238 & 44122 & 90 & 818 & 81322 \\
\hline \hline $0<R_{F}<1$ & 23 & 1818 & 4 & 0 & 52 & 1897 \\
\hline $1 \leq R_{F}<5$ & 29 & 3456 & 2348 & 17 & 274 & 6124 \\
\hline $5 \leq R_{F}<10$ & 2 & 5302 & 4534 & 28 & 262 & 10128 \\
\hline $10 \leq R_{F}<20$ & 0 & 6728 & 7774 & 21 & 171 & 14694 \\
\hline $20 \leq R_{F}<50$ & 0 & 10500 & 14149 & 21 & 59 & 24729 \\
\hline $50 \leq R_{F}$ & 0 & 8434 & 15313 & 3 & 0 & 23750 \\
\hline
\end{tabular}




\begin{tabular}{|r||c|c|c|c|c||r|}
\hline & $\mathrm{C} 4$ & $\mathrm{D} 4$ & $\mathrm{~S} 4$ & $\mathrm{~A} 4$ & $\mathrm{~V} 4$ & \\
\hline \hline frequency & $0.07 \%$ & $44.56 \%$ & $54.26 \%$ & $0.11 \%$ & $1.01 \%$ & frequency \\
\hline \hline $0<\boldsymbol{R}_{\boldsymbol{F}}<1$ & $42.59 \%$ & $5.02 \%$ & $0.01 \%$ & $0.00 \%$ & $6.36 \%$ & $2.33 \%$ \\
\hline $1 \leq \boldsymbol{R}_{\boldsymbol{F}}<5$ & $53.70 \%$ & $9.54 \%$ & $5.32 \%$ & $18.89 \%$ & $33.50 \%$ & $7.53 \%$ \\
\hline $5 \leq \boldsymbol{R}_{\boldsymbol{F}}<10$ & $3.70 \%$ & $14.63 \%$ & $10.28 \%$ & $31.11 \%$ & $32.03 \%$ & $12.45 \%$ \\
\hline $10 \leq \boldsymbol{R}_{\boldsymbol{F}}<20$ & $0.00 \%$ & $18.57 \%$ & $17.62 \%$ & $23.33 \%$ & $20.90 \%$ & $18.07 \%$ \\
\hline $20 \leq \boldsymbol{R}_{\boldsymbol{F}}<50$ & $0.00 \%$ & $28.98 \%$ & $32.07 \%$ & $23.33 \%$ & $7.21 \%$ & $30.41 \%$ \\
\hline $50 \leq \boldsymbol{R}_{\boldsymbol{F}}$ & $0.00 \%$ & $23.27 \%$ & $34.71 \%$ & $3.33 \%$ & $0.00 \%$ & $29.20 \%$ \\
\hline
\end{tabular}

\section{Class groups}

The computation of the class groups had definitely more interesting results than in the totally real case. While in the real case over $90 \%$ of the class groups turned out to be trivial and the maximal class number was only six, we now found class numbers up to 70 . Moreover, more than half of the class groups $(54.4 \%)$ were nontrivial.

The algorithm for computing the class groups was already presented in [3]; see $[7,8]$ for greater details. Hence, we give only a short summary of the method. For each field we begin by computing a superset of generators of the class group. According to a theorem of Zimmert [11] there exists an integral ideal in every ideal class whose norm is bounded by $\sqrt{d_{F}} / 6.792 \leq 10^{3} / 6.792<148$.

For a particular field it is hence sufficient to compute all prime ideals $\mathbf{p}_{1}, \ldots, \mathbf{p}_{v}$ lying over primes $p$ subject to $p \leq 139$. With the help of methods from the geometry of numbers, we then determine sufficiently many relations between those prime ideals $[3,10]$. The relations are listed in a so-called class group matrix:

$$
\mathrm{CGM}:=\left(c_{i, j}\right) \in \mathbb{Z}^{v \times w} \quad\left(w \in \mathbb{Z}^{>0}\right)
$$

where

$$
\forall j \in\{1, \ldots, w\}: \quad \prod_{i=1}^{v} \mathbf{p}_{i}^{c_{i, j}} \text { is a principal ideal. }
$$

Condition (2) is invariant under elementary column operations of CGM. Hence, we compute the lower Hermite normal form of (1) (see [8]). If the resulting matrix is singular, we need more relations, which can be obtained by fast deterministic methods [10]. If the matrix is nonsingular, its determinant is a multiple of the class number; i.e., if the determinant is one we have already proved that $h_{F}=1$. Otherwise, we can delete all rows and columns with diagonal entry 1 without any information being lost. We call the resulting matrix reduced class group matrix. In none of the cases did the rank of the resulting matrix exceed five.

The task of the last step is to derive the class group structure explicitly. The method used is explained for the general case in [7, 8, 2]. We illustrate the procedure by an example.

Let $F:=\mathbb{Q}(\rho)$, where $\rho^{4}+65 \rho^{2}+995=0$. The field discriminant is 398000 , an integral basis is given by $1, \rho,\left(1+\rho^{2}\right) / 7,\left(\rho+\rho^{3}\right) / 7$ and the regulator is $R_{F} \approx 0.9624$. Although Zimmert's bound is 92 , we choose 150 as norm bound for the ideals to be taken under consideration. This is to make the calculation of relations more efficient [10]. We obtain 48 prime ideals over primes below 150 . 
After detecting about 80 relations (by the methods of [3]) we get the following reduced class group matrix:

$$
\left(\begin{array}{lllll}
2 & 0 & 0 & 0 & 0 \\
0 & 2 & 0 & 0 & 0 \\
1 & 1 & 2 & 0 & 0 \\
1 & 0 & 1 & 2 & 0 \\
0 & 1 & 1 & 0 & 2
\end{array}\right) .
$$

Hence, only five prime ideals $\mathbf{p}_{1}, \ldots, \mathbf{p}_{5}$ (with norms $71,11,49,5$, and 4 ) are left and five relations between these ideals: (i) $\mathbf{p}_{1}^{2} \mathbf{p}_{3} \mathbf{p}_{4} \in \mathscr{H}_{F}$, (ii) $\mathbf{p}_{2}^{2} \mathbf{p}_{3} \mathbf{p}_{5} \in \mathscr{H}_{F}$, (iii) $\mathbf{p}_{3}^{2} \mathbf{p}_{4} \mathbf{p}_{5} \in \mathscr{H}_{F}$, (iv) $\mathbf{p}_{4}^{2} \in \mathscr{H}_{F}$, (v) $\mathbf{p}_{5}^{2} \in \mathscr{H}_{F}$, where $\mathscr{H}_{F}$ denotes the set of principal ideals of the maximal order $o_{F}$ of $F$. The class number divides 32 .

For deriving the exact class group structure we need an efficient principal ideal test. As in the totally real case we used the method of Fincke and Pohst [5]; however, for large regulators (i.e., $R_{F}>50$ ) the principal ideal test of Buchmann and Williams [4] turned out to be faster.

In detail, we search for ideals $\mathbf{a}_{1}, \ldots, \mathbf{a}_{\nu}$ which generate $\nu(\leq 5)$ cyclic factors of the class group $\mathrm{Cl}_{F}$ such that

$$
\mathrm{Cl}_{F}=\left\langle\mathbf{a}_{1} \mathscr{H}_{F}\right\rangle \times \cdots \times\left\langle\mathbf{a}_{\nu} \mathscr{H}_{F}\right\rangle
$$

and

$$
\operatorname{ord}\left(\mathbf{a}_{i} \mathscr{H}_{F}\right) \mid \operatorname{ord}\left(\mathbf{a}_{i+1} \mathscr{H}_{F}\right) \quad(1 \leq i<\nu) .
$$

Clearly, the $\mathbf{a}_{i}$ can be determined as power products of $\mathbf{p}_{1}, \ldots, \mathbf{p}_{5}$. Note that we obtain the ideals $\mathbf{a}_{i}$ in reverse order at first.

Starting with $\mathbf{p}_{5}$ and condition (v), we check whether $\mathbf{p}_{5} \in \mathscr{H}_{f}$. The result is negative. Hence, we set $\mathbf{a}_{1} \leftarrow \mathbf{p}_{5}, C \leftarrow\left\langle\mathbf{a}_{1} \mathscr{H}_{F}\right\rangle$ and go on with condition (iv) and $\mathbf{p}_{4}$. We have to compute the least exponent $m>0$ such that $\mathbf{p}_{4}^{m} \mathscr{H}_{F} \in$ $C$. Since we know that $\mathbf{p}_{4}^{2} \in \mathscr{H}_{F}$, we must only check whether $\mathbf{p}_{4} \in \mathscr{H}_{F}$ or $\mathbf{p}_{4} \mathbf{a}_{1} \in \mathscr{K}_{F}$. Both tests yield negative results. Therefore, we enlarge $C$ by setting $\mathbf{a}_{2} \leftarrow \mathbf{p}_{4}$ and $C \leftarrow\left\langle\mathbf{a}_{1} \mathscr{H}_{F}\right\rangle \times\left\langle\mathbf{a}_{2} \mathscr{H}_{F}\right\rangle$. Condition (iii) is already optimal in the sense that $\mathbf{p}_{3}^{1} \mathscr{H}_{F} \in C$ is impossible. Immediately, we can apply the elementary divisor theorem to the lower right $(3 \times 3)$-submatrix of CGM, which yields $\operatorname{diag}(1,2,4)$. Because of necessary row operations we have to modify the generators $\mathbf{a}_{1}, \mathbf{a}_{2}$ via the inverse of the transformation matrix and get $\mathbf{a}_{1} \leftarrow\left(\mathbf{p}_{3} \mathbf{p}_{4}\right)^{-1}, \mathbf{a}_{2} \leftarrow \mathbf{p}_{4}$, and $C \leftarrow\left\langle\mathbf{a}_{1} \mathscr{H}_{F}\right\rangle \times\left\langle\mathbf{a}_{2} \mathscr{H}_{F}\right\rangle$. The class group matrix itself becomes

$$
\left(\begin{array}{llll}
2 & 0 & 0 & 0 \\
0 & 2 & 0 & 0 \\
0 & 0 & 2 & 0 \\
3 & 1 & 0 & 4
\end{array}\right),
$$

yielding the conditions (i') $\mathbf{p}_{1}^{2} \mathbf{a}_{1}^{3} \in \mathscr{H}_{F},\left(\mathrm{ii}^{\prime}\right) \mathbf{p}_{2}^{2} \mathbf{a}_{1} \in \mathscr{H}_{F}$.

Again, condition (ii') is optimal, i.e., $\mathbf{p}_{2}^{1} \notin C$. An application of the elementary divisor theorem to the lower right $(3 \times 3)$-submatrix of the class group matrix yields as (reduced) class group matrix

$$
\left(\begin{array}{lll}
2 & 0 & 0 \\
0 & 2 & 0 \\
6 & 0 & 8
\end{array}\right)
$$


with corresponding ideals $\mathbf{a}_{1} \leftarrow\left(\mathbf{p}_{2}\right)^{-1}$ and $\mathbf{a}_{2} \leftarrow \mathbf{p}_{4}$. In the last step the principal ideal test $\mathbf{p}_{1} \mathbf{a}_{1}^{3} \in \mathscr{H}_{F}$ yields a positive result; i.e., we find a principal ideal generator of the ideal $\mathbf{p}_{1} \mathbf{p}_{2} \mathbf{p}_{4} \mathbf{p}_{5}$ which is equivalent to $\mathbf{p}_{1} \mathbf{a}_{1}^{3}$. Finally, reordering the ideals $\mathbf{a}_{i}$, we obtain the result

$$
\mathrm{Cl}_{F}=\left\langle\mathbf{a}_{1} \mathscr{H}_{F}\right\rangle \times\left\langle\mathbf{a}_{2} \mathscr{H}_{F}\right\rangle
$$

with ideals

$$
\begin{aligned}
& \mathbf{a}_{1}=\mathbf{p}_{4}=11 o_{F}+(5+\rho) o_{F}, \\
& \mathbf{a}_{2}=\mathbf{p}_{2}=5 o_{F}+\rho o_{F}
\end{aligned}
$$

of order 2 and 8 , respectively.

We note that we had to carry out only four principal ideal tests.

The following table describes the occurrence of noncyclic class groups in dependence on the Galois group structure:

\begin{tabular}{|c|c|c|c|c||r|}
\hline C4 & D4 & S4 & A4 & V4 & $\sum$ \\
\hline \hline 26 & 3891 & 994 & 3 & 254 & 5168 \\
\hline $48.15 \%$ & $10.74 \%$ & $2.25 \%$ & $3.33 \%$ & $31.05 \%$ & $6.35 \%$ \\
\hline
\end{tabular}

Before going into detail, we give a survey of the class number distribution:

\begin{tabular}{|c||c|c|c|c|c||r|}
\hline & $\mathrm{C} 4$ & $\mathrm{D} 4$ & $\mathrm{~S} 4$ & $\mathrm{~A} 4$ & $\mathrm{~V} 4$ & $\#$ \\
\hline \hline$\#$ & 54 & 36238 & 44122 & 90 & 818 & 81322 \\
\hline \hline$h_{F}=1$ & 7 & 11841 & 25154 & 13 & 40 & 37055 \\
\hline$h_{F}=2$ & 8 & 9353 & 8784 & 33 & 93 & 18271 \\
\hline$h_{F}=3$ & 0 & 1775 & 2726 & 1 & 53 & 4555 \\
\hline$h_{F}=4$ & 12 & 4769 & 2894 & 32 & 154 & 7861 \\
\hline $5 \leq h_{F}<10$ & 8 & 4293 & 3249 & 7 & 245 & 7802 \\
\hline $10 \leq h_{F}<20$ & 11 & 2902 & 1101 & 4 & 146 & 4164 \\
\hline $20 \leq h_{F}$ & 8 & 1305 & 214 & 0 & 87 & 1614 \\
\hline
\end{tabular}

\begin{tabular}{|c||c|c|c|c|c||r|}
\hline & C4 & D4 & S4 & A4 & V4 & frequency \\
\hline \hline frequency & $0.07 \%$ & $44.56 \%$ & $54.26 \%$ & $0.11 \%$ & $1.01 \%$ & $100 \%$ \\
\hline \hline$h_{F}=-1$ & $12.96 \%$ & $32.68 \%$ & $57.01 \%$ & $14.44 \%$ & $4.89 \%$ & $45.57 \%$ \\
\hline$h_{F}=2$ & $14.81 \%$ & $25.81 \%$ & $19.91 \%$ & $36.67 \%$ & $11.37 \%$ & $22.47 \%$ \\
\hline$h_{F}=3$ & $0.00 \%$ & $4.90 \%$ & $6.18 \%$ & $1.11 \%$ & $6.48 \%$ & $5.60 \%$ \\
\hline$h_{F}=4$ & $22.22 \%$ & $13.16 \%$ & $6.56 \%$ & $35.56 \%$ & $18.83 \%$ & $9.67 \%$ \\
\hline $5 \leq h_{F}<10$ & $14.81 \%$ & $11.85 \%$ & $7.36 \%$ & $7.78 \%$ & $29.95 \%$ & $9.59 \%$ \\
\hline $10 \leq h_{F}<20$ & $20.37 \%$ & $8.01 \%$ & $2.50 \%$ & $4.44 \%$ & $17.85 \%$ & $5.12 \%$ \\
\hline $20 \leq h_{F}$ & $14.81 \%$ & $3.60 \%$ & $0.49 \%$ & $0.00 \%$ & $10.64 \%$ & $1.98 \%$ \\
\hline
\end{tabular}

We conclude with a more detailed survey of the class group structures that occur. The following table shows the frequency of each class group and the corresponding minimal field discriminant (if less than $10^{6}$ ). 


\begin{tabular}{|r|c||c|c|c|c|c||r|}
\hline$h_{F}$ & $C l_{F}$ & $\mathrm{C} 4$ & $\mathrm{D} 4$ & $\mathrm{~S} 4$ & $\mathrm{~A} 4$ & $\mathrm{~V} 4$ & $\sum$ \\
\hline \hline 1 & 1 & $7(125)$ & $11841(117)$ & $25154(229)$ & $13(3136)$ & $40(144)$ & 37055 \\
\hline 2 & 2 & $8(8000)$ & $9353(1872)$ & $8784(2889)$ & $33(4225)$ & $93(1521)$ & 18271 \\
\hline 3 & 3 & - & $1775(3897)$ & $2726(7249)$ & $1(876096)$ & $53(4761)$ & 4555 \\
\hline 4 & 4 & $2(256000)$ & $2698(8000)$ & $2224(11348)$ & $29(15376)$ & $92(9025)$ & 5045 \\
\hline 4 & $2 \times 2$ & $10(18000)$ & $2071(20800)$ & $670(40437)$ & $3(246016)$ & $62(24336)$ & 2816 \\
\hline 5 & 5 & - & $689(12176)$ & $991(13396)$ & - & $40(14161)$ & 1720 \\
\hline 6 & 6 & - & $1459(20025)$ & $805(23297)$ & $1(819025)$ & $54(24025)$ & 2319 \\
\hline 7 & 7 & - & $340(25205)$ & $513(26028)$ & - & $18(45369)$ & 871 \\
\hline 8 & 8 & - & $770(34704)$ & $449(37108)$ & $6(205209)$ & $49(38025)$ & 1274 \\
\hline 8 & $2 \times 2 \times 2$ & $5(136125)$ & $83(187200)$ & $3(589392)$ & - & $12(112896)$ & 103 \\
\hline 8 & $2 \times 4$ & $3(210125)$ & $723(13500)$ & $228(109008)$ & - & $61(17424)$ & 1015 \\
\hline 9 & 9 & - & $195(36513)$ & $253(46453)$ & - & $5(112225)$ & 453 \\
\hline 9 & $3 \times 3$ & - & $34(127813)$ & $7(205609)$ & - & $6(103041)$ & 47 \\
\hline 10 & 10 & $7(44217)$ & $616(48528)$ & $263(77648)$ & $2(494209)$ & $16(127449)$ & 904 \\
\hline 11 & 11 & - & $165(54025)$ & $164(67581)$ & - & $7(251001)$ & 336 \\
\hline 12 & 12 & - & $403(67648)$ & $137(106956)$ & - & $17(61504)$ & 557 \\
\hline 12 & $2 \times 6$ & - & $376(108225)$ & $37(226064)$ & - & $33(76176)$ & 446 \\
\hline 13 & 13 & - & $142(64576)$ & $100(115708)$ & - & $5(303601)$ & 247 \\
\hline 14 & 14 & - & $311(78912)$ & $102(118548)$ & - & $9(126025)$ & 422 \\
\hline 15 & 15 & - & $126(83008)$ & $68(114460)$ & - & $10(99856)$ & 204 \\
\hline 16 & 16 & - & $182(104512)$ & $53(134036)$ & $2(529984)$ & $7(308025)$ & 244 \\
\hline 16 & $2 \times 2 \times 4$ & $4(722000)$ & $27(342000)$ & - & - & $5(176400)$ & 36 \\
\hline
\end{tabular}

\begin{tabular}{|c|c||c|c|c|c|c||r|}
\hline$h_{F}$ & $C l_{F}$ & $\mathrm{C} 4$ & $\mathrm{D} 4$ & $\mathrm{~S} 4$ & $\mathrm{~A} 4$ & $\mathrm{~V} 4$ & $\sum$ \\
\hline \hline 16 & $2 \times 8$ & - & $203(124992)$ & $27(224568)$ & - & $25(278784)$ & 255 \\
\hline 16 & $4 \times 4$ & - & $32(334080)$ & $5(534784)$ & - & $4(176400)$ & 41 \\
\hline 17 & 17 & - & $83(120025)$ & $54(173164)$ & - & - & 137 \\
\hline 18 & 18 & - & $158(135025)$ & $42(183564)$ & - & $1(919681)$ & 201 \\
\hline 18 & $3 \times 6$ & - & $16(223025)$ & - & - & $6(121104)$ & 22 \\
\hline 19 & 19 & - & $62(125137)$ & $49(173713)$ & - & $1(870489)$ & 112 \\
\hline 20 & 20 & $2(256000)$ & $151(180025)$ & $31(294813)$ & - & $14(141376)$ & 198 \\
\hline 20 & $2 \times 10$ & $4(392000)$ & $141(155664)$ & $6(455749)$ & - & $10(184041)$ & 161 \\
\hline 21 & 21 & - & $45(189025)$ & $23(155444)$ & - & $5(152881)$ & 73 \\
\hline 22 & 22 & -- & $102(196672)$ & $24(292517)$ & - & - & 126 \\
\hline 23 & 23 & - & $32(231025)$ & $18(189816)$ & - & - & 50 \\
\hline 24 & 24 & - & $88(218176)$ & $14(331125)$ & - & $8(189225)$ & 110 \\
\hline 24 & $2 \times 2 \times 6$ & - & $11(383625)$ & - & - & $1(853776)$ & 12 \\
\hline 24 & $2 \times 12$ & - & $70(235152)$ & $3(793517)$ & - & $6(336400)$ & 79 \\
\hline 25 & 25 & - & $38(264256)$ & $8(389620)$ & - & - & 46 \\
\hline 25 & $5 \times 5$ & - & $1(946525)$ & - & - & $2(373321)$ & 3 \\
\hline 26 & 26 & - & $77(233536)$ & $15(340008)$ & - & $1(912025)$ & 93 \\
\hline 27 & 27 & - & $26(248896)$ & $11(340741)$ & - & -- & 37 \\
\hline 27 & $3 \times 9$ & - & - & - & - & $1(277729)$ & 1 \\
\hline 28 & 28 & - & $46(290448)$ & $8(746684)$ & - & - & 54 \\
\hline 28 & $2 \times 14$ & - & $39(344025)$ & $4(412812)$ & - & $3(725904)$ & 46 \\
\hline 29 & 29 & - & $17(298537)$ & $5(464212)$ & - & - & 22 \\
\hline 30 & 30 & - & $52(279616)$ & $3(545013)$ & - & $6(404496)$ & 61 \\
\hline 31 & 31 & - & $18(475025)$ & $5(549361)$ & - & - & 23 \\
\hline 32 & 32 & - & $36(411408)$ & $5(497268)$ & - & - & 41 \\
\hline 32 & $2 \times 2 \times 8$ & - & $1(922625)$ & - & - & - & 1 \\
\hline 32 & $2 \times 16$ & - & $22(480528)$ & $2(766125)$ & - & $6(439569)$ & 30 \\
\hline 32 & $4 \times 8$ & - & $6(723600)$ & - & - & $3(608400)$ & 9 \\
\hline 33 & 33 & - & $17(334668)$ & $8(402300)$ & - & $2(588289)$ & 27 \\
\hline 34 & 34 & $2(594473)$ & $30(384064)$ & $2(761013)$ & - & - & 34 \\
\hline 35 & 35 & - & $24(329141)$ & $6(547757)$ & - & $1(851929)$ & 31 \\
\hline 36 & 36 & - & $25(398400)$ & $1(645004)$ & - & $1(990025)$ & 27 \\
\hline 36 & $2 \times 18$ & - & $16(540736)$ & $2(880884)$ & - & - & 18 \\
\hline 36 & $3 \times 12$ & - & - & - & - & $3(483025)$ & 3 \\
\hline 37 & 37 & - & $11(405568)$ & $1(762808)$ & - & - & 12 \\
\hline 38 & 38 & - & $28(494352)$ & $2(643897)$ & - & - & 30 \\
\hline 39 & 39 & - & $13(491584)$ & - & - & - & 13 \\
\hline 40 & 40 & - & $21(504600)$ & $1(947348)$ & - & $5(511225)$ & 27 \\
\hline & & & & & & & \\
\hline
\end{tabular}




\begin{tabular}{|r|c||r|c|c|c|c||r|}
\hline$h_{F}$ & $C l_{F}$ & $\mathrm{C} 4$ & $\mathrm{D} 4$ & $\mathrm{~S} 4$ & $\mathrm{~A} 4$ & $\mathrm{~V} 4$ & $\Sigma$ \\
\hline \hline 40 & $2 \times 2 \times 10$ & - & - & - & - & $1(906304)$ & 1 \\
\hline 40 & $2 \times 20$ & - & $11(549000)$ & - & - & $1(906304)$ & 12 \\
\hline 41 & 41 & - & $9(520489)$ & $2(727656)$ & - & - & 11 \\
\hline 42 & 42 & - & $11(639561)$ & $1(818901)$ & - & $1(570025)$ & 13 \\
\hline 43 & 43 & - & $9(684025)$ & $1(650264)$ & - & - & 10 \\
\hline 44 & 44 & - & $12(424000)$ & - & - & - & 12 \\
\hline 44 & $2 \times 22$ & - & $2(861025)$ & - & - & - & 2 \\
\hline 45 & 45 & - & $2(901184)$ & - & - & - & 2 \\
\hline 46 & 46 & - & $10(589849)$ & - & - & - & 10 \\
\hline 47 & 47 & - & $3(783025)$ & - & - & - & 3 \\
\hline 48 & 48 & - & $7(673081)$ & - & - & $2(577600)$ & 9 \\
\hline 48 & $2 \times 24$ & - & $1(902025)$ & - & - & $1(853776)$ & 2 \\
\hline 49 & 49 & - & $3(774208)$ & - & - & - & 3 \\
\hline 50 & 50 & - & $5(812304)$ & - & - & - & 5 \\
\hline 50 & $5 \times 10$ & - & - & - & - & $1(678976)$ & 1 \\
\hline 51 & 51 & - & $4(654373)$ & - & -- & - & 4 \\
\hline 52 & 52 & - & $1(964368)$ & - & - & - & 1 \\
\hline 52 & $2 \times 26$ & - & $4(871488)$ & - & - & - & 4 \\
\hline 53 & 53 & - & - & $1(833044)$ & - & - & 1 \\
\hline 55 & 55 & - & $1(920337)$ & - & - & - & 1 \\
\hline 56 & 56 & - & $1(958528)$ & - & - & $1(874225)$ & 2 \\
\hline 57 & 57 & - & $1(929713)$ & -- & - & - & 1 \\
\hline 60 & 60 & - & $1(849660)$ & - & - & - & 1 \\
\hline 60 & $2 \times 30$ & - & - & - & - & $1(846400)$ & 1 \\
\hline 64 & 64 & - & $1(654400)$ & - & - & - & 1 \\
\hline 64 & $8 \times 8$ & - & $1(790920)$ & - & - & - & 1 \\
\hline 68 & 68 & - & $1(769600)$ & - & - & - & 1 \\
\hline 70 & 70 & - & - & $1(958616)$ & - & - & 1 \\
\hline \hline$\sum$ & & 54 & 36238 & 44122 & 90 & 818 & 81322 \\
\hline
\end{tabular}

All computations were done on Apollo workstations DN3000 and DN4500 (CPU Motorola 68020/68030). We used the number-theoretic program library KANT, which is developed in Düsseldorf [9]. All data can be obtained from the authors.

\section{ACKNOWLEDGMENT}

We thank the referee for several useful suggestions.

\section{BIBLIOGRAPHY}

1. J. Buchmann, The computation of the fundamental unit in totally complex quartic orders, Math. Comp. 48 (1987), 39-54.

2. J. Buchmann and M. Pohst, On the complexity of computing class groups of algebraic number fields, Applied Algebra, Algebraic Algorithms and Error-Correcting Codes, Proc. AAECC-6, Rome 1988, Lecture Notes in Comput. Sci., vol. 357, Springer-Verlag, 1989, pp. 122-131.

3. J. Buchmann, M. Pohst, and J. v. Schmettow, On the computation of unit groups and class groups of totally real quartic fields, Math. Comp. 53 (1989), 387-397.

4. J. Buchmann and H. C. Williams, On principal ideal testing in algebraic number fields, J. Symbolic Comput. 4 (1987), 11-19.

5. U. Fincke and M. Pohst, $A$ procedure for determining algebraic integers of given norm, Proc. EUROCAL 83, Lecture Notes in Comput. Sci., vol. 162, Springer-Verlag, 1983, pp. 194-202. 
6. D. Ford, Enumeration of totally complex quartic fields of small discriminant, Computational Number Theory (A. Pethö, M. E. Pohst, H. C. Williams, and H. G. Zimmer, eds.), de Gruyter, 1991, pp. 129-138.

7. M. Pohst and H. Zassenhaus, Über die Berechnung von Klassenzahlen und Klassengruppen algebraischer Zahlkörper, J. Reine Angew. Math. 361 (1985), 50-72.

8. __ Algorithmic algebraic number theory, Cambridge Univ. Press, New York, 1989.

9. J. Graf v. Schmettow, KANT-a tool for computations in algebraic number fields, Computational Number Theory (A. Pethö, M. E. Pohst, H. C. Williams, and H. G. Zimmer, eds.), de Gruyter, 1991, pp. 321-330.

10. __ Beiträge zur Klassengruppenberechnung, Dissertation, Düsseldorf, 1991.

11. R. Zimmert, Ideale kleiner Norm in Idealklassen und eine Regulatorabschätzung, Invent. Math. 62 (1981), 367-380.

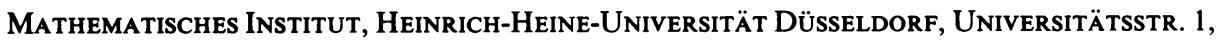
4000 DÜSSELDORF 1, GERMANY

E-mail address: pohst@ze8.rz.uni-duesseldorf.de 